\title{
Hypercalcemia in Patients Treated with Oral Bisphosphonates for Tumor-Induced Osteolysis
}

\author{
Michał Holecki ${ }^{1^{*}}$, Anna Skorupa ${ }^{2}$, Jan Duława ${ }^{1}$, Jerzy Chudek $^{3}$ \\ ${ }^{1}$ Department of Internal Medicine and Metabolic Diseases, Medical University of Silesia, Katowice, Poland; ${ }^{2}$ Department of \\ Anaesthesiology and Intensive Care, Medical University of Silesia, Katowice, Poland; ${ }^{3}$ Department of Pathophysiology, \\ Medical University of Silesia, Katowice, Poland
}

\begin{abstract}
Citation: Holecki M, Skorupa A, Duława J, Chudek J. Hypercalcemia in Patients Treated with Oral Bisphosphonates for Tumor-Induced Osteolysis. OA Maced J Med Sci. 2013 Dec 15; 1(1):54-58.

http://dx.doi.org/10.3889/oamjms.2013.011

Key words: bisphosphonates; hypercalcemia; malignancy; osteolysis; bone resorption.

"Correspondence: Dr. Michał Holecki. Medical University of Silesia, Department of Internal Medicine and Metabolic Diseases, Ziolowa 45/47, Katowice 40-635, Poland. E-Mail: holomed@poczta.onet.pl

Received: 22-Sep-2013; Revised: 24-Sep2013; Accepted: 18-Oct-2013; Online first: 05-Nov-2013

Copyright: $\odot 2013$ Holecki M. This is an openaccess article distributed under the terms of the Creative Commons Attribution License, which permits unrestricted use, distribution, and reproduction in any medium, provided the original author and source are credited.

Competing Interests: The authors have declared that no competing interests exist.
\end{abstract}

\begin{abstract}
Objective: Hypercalcemia as the consequence of an excessive bone resorption is a common complication in patients with cancer. The aim of the study was to analyze the prevalence of hypercalcemia in patients with tumor-induced osteolysis starting therapy with bisphosphonates.

Methods: The questionnaire-based survey (data collected during three consecutive examinations within a 3-month period) was conducted among 1,450 patients treated with bisphosphonates for tumor-induced osteolysis.

Results: Hypercalcemia was found in $8.7 \%$ respondents starting the treatment with bisphosphonates. The most common cause of malignancy-associated hypercalcemia was prostate cancer, multiple myeloma and breast cancer. On the other hand, hypercalcemia was the most prevalent among patients with multiple myeloma, metastatic cancer of an unknown primary origin and bladder cancer. Metastases were reported in 342 patients, while pathological fractures in 37. The normalization of calcium level was obtained in $91.4 \%$ of the patients treated with bisphosphonates, mostly clodronate. During the bisphosphonate therapy, pathological fractures occurred in $4.6 \%$ of patients and the percentage of the patients reporting bone pain decreased from $79.9 \%$ to $30.9 \%$
\end{abstract}

Conclusion: Multiple myeloma, prostate and breast cancer are the most common causes of hypercalcemia of malignancy in patients with tumor-induced osteolysis starting therapy with bisphosphonates.

\section{Introduction}

Hypercalcemia resulting from an excessive bone resorption is a common complication in patients with advanced cancer, and the malignancy is the most common cause of hypercalcemia in clinical practice (approximately $70 \%$ of cases) [1]. There are three major cancers particularly contributing to the development of hypercalcemia: multiple myeloma (MM) (in approximately $20-40 \%$ of patients), T-cell lymphoma (about $50 \%$ of patients) and breast cancer (in $20-40 \%$ of cases) [2-4]. The primary mechanism of hypercalcemia in the patients with cancer is an increased bone resorption resulting from tumor secretion of cytokines, PTH-related peptide (PTHrp) and calcitriol [5]. Bone pain is the most common clinical sign of increased tumor-induced osteolysis, reported by approximately $60 \%$ of patients [6].

The use of bisphosphonates is the treatment of choice in patients with tumor-induced osteolytic hypercalcemia. The drugs bind to the surface of hydroxyapatite, inhibit osteoclast-mediated bone osteolysis (thus decreasing calcium release and formation), inhibit maturation of osteoclasts (by inducing their apoptosis), reduce the loss of bone mineral mass and the risk of bone fractures and have an analgesic effect [7-9]. 
The aim of the study was to analyze the prevalence of hypercalcemia in patients with tumorinduced osteolysis starting therapy with bisphosphonates.

\section{Materials and Methods}

The study, performed in the years 2009-2010, consists of a survey involving 172 physicians who agreed to participate in the study of about 300 eligible working in oncologic and pain management outpatient clinics throughout the country. Specialists were recruited by medical representatives who distributed the questionnaires, being obliged not to advertise any specific bisphosphonate during the visit. The participating physicians were asked to recruit among their patients diagnosed with cancer the ones treated with bisphosphonates due to tumor-induced osteolysis established on the basis of the latest available X-ray imaging with serum calcium level examined prior to the initiation of the therapy with primary hyperparathyroidism being the only exclusion criterion. The physician filling the questionnaire declared that have complete insight into medical records of the patient. This declaration was not verified.

The approval of the ethical committee was not required as the questionnaire-based survey was based on a routine follow up of clinical patients, no patient identification was recorded and thus the study did not fulfill the criterion of a medical experiment.

The participating physicians were asked to complete the 3-part questionnaire on the basis of medical records (excluding the patients' personal details), during three consecutive examinations within a 3-month period. The first part of the questionnaire (completed during the first examination) included demographic data (sex, age, place of residence) and information about: the type of cancer, the clinical course of the disease before the implementation of bisphosphonate therapy (tumor-induced osteolysis, pathological fractures due to metastasis, hypercalcemia of malignancy), the duration of the treatment, the dose and type of bisphosphonate used, the serum calcium level at the time when hypercalcemia was diagnosed, the occurrence of cancer-related bone pain, pathological fractures that occurred during bisphosphonate treatment and the history of any previous treatment with intravenous bisphosphonates. Serum total calcium level over 10.5 $\mathrm{mg} / \mathrm{dl}$ was defined as hypercalcemia. Additionally, the participating physicians were asked to check if any previous bisphosphonate treatment resulted in the normalization of calcium level. The second and third part of the questionnaire were completed during a 3month follow-up period. This time the participating physicians were asked to record the data related to the therapeutic effect of the applied treatment, e.g.: the occurrence of newly diagnosed pathologic bone fractures or hypercalcemia, the persistence of hypercalcemia, the occurrence of self-reported bone pain and the initiation of the treatment with calcium and vitamin $\mathrm{D}$. The presence of bone pain defined as a tenderness of the skeleton, worsening on palpation, with characteristic enhancement during the night, was assessed on the basis on patients' complaints during the last week prior to examination.

Bisphosphonates were mainly used orally. Only in 37 hypercalcemic (26.6\%) and 204 normocalcemic (15.3\%) patients bisphosphonates were initially used intravenously. The compliance to orally given bisphosphonates was measured by the frequency of missing drug doses between consecutive examinations reported by patients or patients' guardian.

Of the planned recruitment of 5,000 patients diagnosed with tumor-induced osteolysis treated with bisphosphonates, only 1,495 subjects $(29.9 \%$ effectiveness) were enrolled due to lower than initially planned effectiveness of participating physicians recruitment.15 subjects treated with bisphosphonates for osteoporosis were excluded from the analysis.

All data are expressed as percentages or means with standard deviations. The analyses were performed using the STATISTICA 10.0 PL (StatSoft Polska, Kraków, Poland) and MedCalc v. 14.4.4.0 (Software, Mariakerke, Belgium) software. The $x^{2}$ test and the logistic regression analysis were applied for the calculation of a relative risk $(R R)$ and their $95 \%$ confidence intervals $(\mathrm{Cl})$. A p value less than 0.05 was considered statistically significant.

\section{Results}

\section{Characteristic of the study group}

The detailed characteristics of the study group are shown in Table 1. Hypercalcemia of malignancy was diagnosed in 129 (8.7\%) of 1480 respondents (in $32.6 \%$ of patients with multiple myeloma, $23.1 \%$ of patients with metastatic cancer of unknown primary origin and in $16.7 \%$ of patients with bladder cancer). The most common cause of hypercalcemia in the group was prostate cancer $(38.0 \%)$, multiple myeloma $(22.5 \%)$ and breast cancer $(20.9 \%)$ - Figure 1 . The mean serum calcium level in the hypercalcemic patients at the time of the diagnosis was 3.06 (2.973.15) $\mathrm{mmol} / \mathrm{l}$.

Metastases were reported in 342 , while pathological fractures in 37 patients $(23.1 \%$ and $2.5 \%$ of the cases respectively). Pathological bone fractures were more frequent among the patients with metastatic disease $(9.8 \%$ vs. $2.2 \%$ in the patients without metastasis, $\mathrm{p}<0.001)$. Bone pain was reported by $79.9 \%$ of the patients with cancer, more frequently in the patients with metastases $(87.8 \%$ vs. $77.5 \%$ without metastasis), but with a similar frequency in patients with and without a history of bone fractures 
and in those with and without hypercalcemia. Hypercalcemia was equally frequent in the patients with and without diagnosed metastases $7.1 \% \mathrm{vs}$. $7.6 \%$ (after exclusion of the patients with multiple myeloma) and unrelated to bone pain.

Table 1: Characteristics of study group $(n=1,480)$. Age $[\mathrm{n},(\%)]$

\begin{tabular}{lr}
$18-40$ years & $13(0.9)$ \\
$41-60$ years & $459(31.0)$ \\
$>60$ years & $1,008(68.1)$ \\
Sex [men (\%)] & $892(60.3)$ \\
Malignant disease [n] & \\
Prostate cancer & 613 \\
Breast cancer & 468 \\
Bronchial cancer & 90 \\
Multiple myeloma & 89 \\
Renal cancer & 81 \\
Colon cancer & 50 \\
Bladder cancer & 18 \\
Cervical / endometrial cancer & 16 \\
Disseminated cancer of unknown primary origin & 13 \\
Others & 42 \\
Period of treatment due to cancer [months] & $34 \pm 30$ \\
Duration of bisphosphonate treatment [months] & $13 \pm 13$ \\
Type of bisphosphonate [n,(\%)] & \\
Alendronate & $12(0.8)$ \\
Clodronate & $1,421(96.0)$ \\
lbandronate & $2(0.1)$ \\
Unspecified & $45(3.1)$ \\
Hypercalcemia [n, $(\%)]$ & $129(8.7)$ \\
Diagnosed metastases [n, $(\%)]$ & $342(23.1)$ \\
Pathological fractures [n, $(\%)]$ & $37(2.5)$ \\
Concomitant osteoporosis [n, $(\%)]$ & $19(1.3)$ \\
\hline
\end{tabular}

The mean duration of the bisphosphonate therapy was $13 \pm 13$ months, with a mean treatment for cancer of at $34 \pm 30$ months. Clodronate was the most commonly used bisphosphonate (96.0\%). Its dose used in the patients without hypercalcemia was $1422 \mathrm{mg}$ (1324-1519), and was significantly lower than the dose used because of hypercalcemia $1504 \mathrm{mg}(1445-1563)(p<0.001)$.

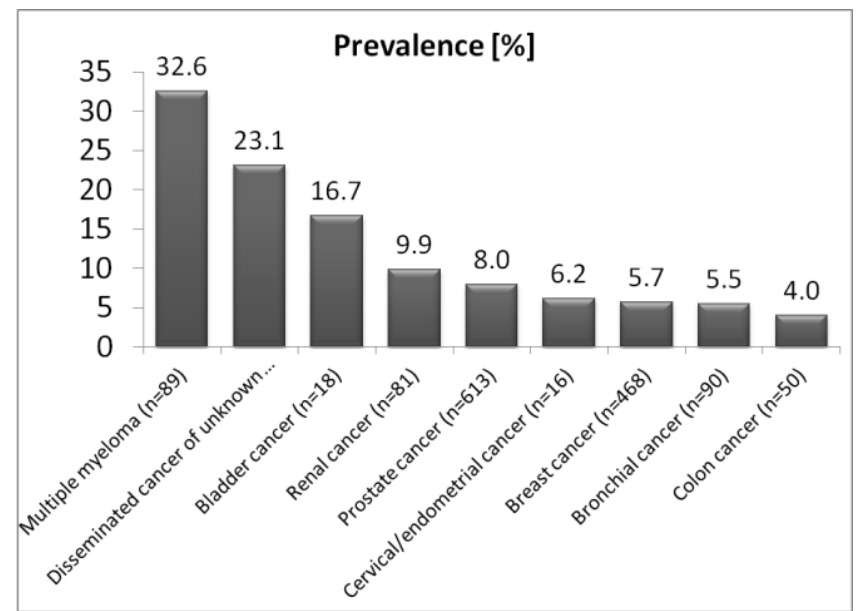

Figure 1: The prevalence of hypercalcemia according to the type of cancer.

\section{Effectiveness of the treatment}

Two consecutive examinations were performed on 1477 and 1476 patients. The compliance with bisphosphonate therapy was $99.4 \%$. The normal range of calcium level was reached in 127 out of 139 hypercalcemic patients $(91.4 \%$ of the patients treated with bisphosphonates). In 37 hypercalcemic $(26.6 \%)$ and 204 normocalcemic $(15.3 \%)$ patients bisphosphonates were initially used intravenously. During the follow-up, bone fracture occurred in 67 patients $(4.6 \%)$, despite the treatment with bisphosphonates in 7 patients with hypercalcemia $(5.0 \%)$ and 60 without hypercalcemia (4.5\%), in $6.3 \%$ of the patients without any previous pathologic bone fractures and $4.8 \%$ without any previous fractures.

During the follow-up, the number of patients reporting bone pain decreased from $79.9 \%$ to $30.9 \%$ $(p<0.001)$. Bone pain remained more common in the patients with a pathological fracture $(50.3 \%$ vs. $28.2 \%$, $\mathrm{p}<0.001), \mathrm{RR}=2.24(1.73-2.92)$.

\section{Discussion}

Hypercalcemia is relatively common in patients with cancer. Osteolytic metastases account for approximately $20 \%$ of cases of hypercalcemia of malignancy [10]. The induction of local osteolysis by tumor cells is common with some solid tumors (eg. breast cancer) that metastasize to bone and with multiple myeloma [11-12]. The bone destruction observed in osteolytic metastases is primarily mediated by osteoclasts, but not by a direct effect of tumor cells [13]. The present study was not designed to assess the prevalence of tumor-induced osteolysis and hypercalcemia of malignancy, but only the incidence of bone complications found in the patients treated with oral bisphosphonates. Thus, on the basis of this study one can only estimate the incidence of hypercalcemia in the patients with tumor-induced osteolysis. Multiple myeloma (MM) was found to be the most common cause for hypercalcemia in the analyzed group of the patients diagnosed with cancer $(32.6 \%)$. Our observation is consistent with the results of another study indicating the presence of this pathology in $28 \%$ of the patients diagnosed with MM [14]. Tumor-induced osteolytic hypercalcemia is often present in the patients with metastatic cancer of unknown primary origin $(23.1 \%)$, thus reflecting its advanced clinical stage. Somewhat surprising is the high percentage of the patients with malignant hypercalcemia in the group of patients with bladder cancer (up to $16.7 \%$ ) which was not a common cause of hypercalcemia in our study group. Previously published studies did not indicate such a high incidence of hypercalcaemia in the patients with bladder cancer. According to the Japanese authors, the incidence was approximately $1.9 \%$ [15]. The reason for these discrepancies is probably a relatively late occurrence of osteolytic metastases of bladder cancer to bone.

Hypercalcemia of malignancy caused by osteolysis was also observed in the patients with renal, cervix / uterus, prostate, breast, bronchial and colon cancers (Figure 1). Bisphosphonates are unique in the treatment of metastatic bone disease as it is a 
bone-targeted therapy. Its effectiveness in lowering the calcium level is not easy to evaluate, as the normalization of calcaemia can only be temporary. In our study, bisphosphonates (clodronate mainly) decreased the calcium serum level in $91.4 \%$ of cases. This result is in consistence with the results of metaanalysis performed by Sanders et al. where the efficacy was evaluated to be over $70 \%$ [16]. Bisphosphonates are also supposed to prevent the occurrence of hypercalcemia in the patients with tumor-induced osteolysis. In the patients with MM, Laakso et al., found clodronate (at a dose of 2.4 $\mathrm{g} /$ day) to be protective against hypercalcemia, as well as against the vertebral and non-vertebral fractures [17]. Bisphosphonates have also been effective in the treatment of bone metastases from other solid tumors, including breast, lung, prostate, kidney and colorectal [18]. In the present study, hypercalcemia was reported only in $0.8 \%$ of patients during the treatment with clodronate. Additionally, very early studies have suggested that the use of clodronate may be protective against fractures both in patients with breast cancer and in patients with multiple myeloma $[18,19]$. These observations were finally proved, however intravenous therapy seemed to be more effective than the oral one. According to the metaanalysis performed by Pavlakis et al. zolendronic acid, pamidronate and ibandronate reduced the bone complications in patients with breast cancer by $41 \%$, $33 \%$ and $18 \%$, respectively [20]. Recently published data by Terpos et al. showed a similar protective effect of bisphosphonates in patients with MM [21]. The natures of our study, without a placebo-treated control group, preclude the assessment of the boneprotective effect of bisphosphonate therapy. In our study, fractures were recorded in $4.6 \%$ of the patients treated with bisphosphonates during the follow-up.

One of the most common complaints made by the patients with skeletal metastases is intractable bone pain. It is postulated that the factors produced by tumor cells, as well as the molecules released by bone remodeling, stimulate pain receptors in bone [22]. According to Clines et al bisphosphonates can reduce the intensity of pain, stimulating the molecules in the microenvironment surrounding metastatic tumor cells [5]. There are some other data that complement our knowledge about a possible anti-tumor activity of bishosphonates. They were found to have direct effects on tumor cells aiding the reduction of tumor growth and its invasiveness, as well as helping induce apoptosis [23-25]. Bisphosphonates may also reduce the adhesion of tumor cells to bone and inhibit angiogenesis [26-27].

The evaluation of bone pain in our study group showed a decrease in the number of patients reporting this problem from $79.9 \%$ to $30.9 \%$. It is consistent with the result of other authors, who showed an analgesic effect of bisphosphonates, in the patients with both MM and prostate cancer [28-29]. However, it must be pointed out that the effectiveness of bisphosphonate is limited when the progression of cancers (increased tumor-induced osteolysis and metastases) is observed. In our study, bone pain remained more common in patients with pathological fractures.

Our study has several limitations, mostly related to the study design and the enrollment of a highly heterogeneous group of patients. The power of statistical analysis was reduced by the lower than initially planned number of participating physicians. The incidence of hypercalcemia was reported by physicians and not controlled in a central laboratory. Moreover, we cannot exclude the selection bias related to the non-included patients with a short life expectancy. Thus our study does not fit in well with the ongoing debate whether bisphosphonates for tumor-induced osteolysis should be administered early in the course of the disease and if they have a beneficial effect on survival. The analysis of the effect of bisphosphonates on bone pain is limited as the treatment with analgesics was not recorded. Therefore, we cannot exclude that in many cases the escalation of analgesics was the cause for the pain relief, as the data concerning this medication (nonsteroid anti inflammatory drug, opioids and coanalgesics) were not collected.

Despite all these limitations our study reflects the prevalence of hypercalcemia in patients with tumor-induced osteolysis not restricted to selected type of malignant disease.

In a conclusion, multiple myeloma, prostate and breast cancer are the most common causes of hypercalcemia of malignancy in patients with tumorinduced osteolysis starting therapy with bisphosphonates.

\section{Acknowledgement}

The study was carried out as a research project of Europharma M. Rachtan Sp. z o. 0. supported by a scientific grant of Polska.

\section{References}

1. Stewart AF. Clinical practice. Hypercalcemia associated with cancer. N Engl J Med. 2005;352:373-9.

2. Mundy GR. Pathogenesis of hypercalcemia of malignancy. Clin Endocrinol (Oxf). 1985;23:705-4.

3. Bunn PA Jr, Schechter GP, Jaffe E, et al. Clinical course of retrovirus-associated adult T-cell lymphoma in the United States. N Engl J Med. 1983;309:257-64.

4. Coleman RE, Rubens RD. The clinical course of bone metastases from breast cancer. Br J Cancer. 1987;55:61-6.

5. Clines GA, Guise TA. Hypercalcaemia of malignancy and basic research on mechanisms responsible for osteolytic and osteoblastic metastasis to bone. Endocr Relat Cancer. 2005;12:549-83.

6. Kyle RA, Gertz MA, Witzig TE, et al. Review of 1027 patients with newly diagnosed multiple myeloma. Mayo Clin Proc. 2003;78:2133. 
7. Croucher PI, De Hendrik R, Perry MJ, et al. Bisphosphonates in multiple myeloma. Cochrane Database Syst Rev. 2002;3:CD003188.

8. Gordon $\mathrm{S}$, Helfrich $\mathrm{MH}$, Sati $\mathrm{HI}$, et al. Pamidronate causes apoptosis of plasma cells in vivo in patients with multiple myeloma. Br J Haematol. 2002;119:475-83.

9. Coleman RE. Bisphosphonates in breast cancer. Ann Oncol 2005;16:687-95.

10. Horwitz MJ, Stewart AF. Hypercalcemia associated with malignancy. In: Primer on the Metabolic Bone Diseases and Disorders of Mineral Metabolism. American Society of Bone and Mineral Research. 2006:195-199.

11. Francini G, Petrioli R, Maioli E, et al. Hypercalcemia in breast cancer. Clin Exp Metastasis. 1993;11:359-67.

12. Mundy GR. Metastasis to bone: causes, consequences and therapeutic opportunities. Nat Rev Cancer. 2002;2:584-93.

13. Quinn JM, Matsumura Y, Tarin D, McGee J, Athanasou N. Cellular and hormonal mechanisms associated with malignant bone resorption. Lab Invest. 1994;71:465-71.

14. Camp NJ, Werner TL, Cannon-Albright LA. Familial myeloma. N Engl J Med. 2008; 359: 1734-5.

15. Higashi Y, Kawamura J, Kanamaru H, Kakehi Y, Yoshida O. 20 cases of hypercalcemia associated with urogenital malignancies. Hinyokika Kiyo. 1984; 30: 599-607.

16. Saunders $Y$, Ross JR, Broadley KE, Edmonds PM, Patel S. Steering Group. Systematic review of bisphosphonates for hypercalcaemia of malignancy. Palliat Med. 2004;18:418-31.

17. Laakso M, Lahtinen R, Virkkunen $P$, Elomaa I. Subgroup and cost-benefit analysis of the Finnish multicentre trial of clodronate in multiple myeloma. Br J Haematol. 1994;87:725-9.

18. Elomaa I, Blomqvist C, Porkka L, Lamberg-Allardt C, Borgström $\mathrm{GH}$. Treatment of skeletal disease in breast cancer: a controlled clodronate trial. Bone. 1987;8(Suppl 1):53-56.

19. Clemens MR, Fessele K, Heim ME. Multiple myeloma: effect of daily dichloromethylene bisphosphonate on skeletal complications. Ann Hematol. 1993;66:141-6.

20. Pavlakis N, Schmidt R, Stockler M. Bisphosphonates for breast cancer. Cochrane Database Syst Rev. 2005;3:CD003474.

21.Terpos E, Dimopoulos MA, Berenson J. Established role of bisphosphonate therapy for prevention of skeletal complications from myeloma bone disease. Crit Rev Oncol Hematol. 2011;77(Suppl 1):13-23.

22. Mantyh PW, Clohisy DR, Koltzenburg M, Hunt S. Molecular mechanisms of cancer pain. Nature Reviews Cancer. 2002;2:2019.

23. Lee YP, Schwarz EM, Davies M, et al. Use of zoledronate to treat osteoblastic versus osteolytic lesions in a severecombinedimmunodeficient mouse model. Cancer Research. 2002;62:556470 .

24. Derenne S, Amiot M, Barille $S$, et al. Zoledronate is a potent inhibitor of myeloma cell growth and secretion of IL-6 and MMP-1 by the tumoral environment. Journal of Bone and Mineral Research. 1999;14:2048-56.

25. Senaratne SG, Pirianov G, Mansi JL, Arnett TR, Colston KW. Bisphosphonates induce apoptosis in human breast cancer cell lines. British Journal of Cancer. 2000;82:1459-68.

26. Boissier S, Magnetto S, Frappart L, et al. Bisphosphonates inhibit prostate and breast carcinoma cell adhesion to unmineralized and mineralized bone extracellular matrices. Cancer Research. 1997;57:3890-4.

27. Fournier $\mathrm{P}$, Boissier $\mathrm{S}$, Filleur $\mathrm{S}$, et al. Bisphosphonates inhibit angiogenesis in vitro and testosterone-stimulated vascular regrowth in the ventral prostate in castrated rats. Cancer Research. 2002;62:6538-44.

28. Lahtinen $R$, Laakso $M$, Palva I, Virkkunen $P$, Elomaa I. Randomised, placebo-controlled multicentre trial of clodronate in multiple myeloma. Finnish Leukaemia Group. Lancet. 1992; 340:1049-52.

29. Yuen KK, Shelley M, Sze WM, Wilt T, Mason MD. Bisphosphonates for advanced prostate cancer. Cochrane Database Syst Rev. 2006;4:CD006250. 